\title{
Cancer-associated carbohydrate antigens for clinical diagnostic markers - its effectiveness and limitations
}

\author{
Yoshinori Inagaki*, Peipei Song, Wei Tang, Norihiro Kokudo \\ Hepato-Biliary-Pancreatic Surgery Division, Department of Surgery, Graduate School of Medicine, The University of Tokyo, \\ Tokyo, Japan.
}

\begin{abstract}
Summary Cancer cells express various aberrant glycoconjugates. Several kinds of carbohydrate antigens have been used for the serological tumor markers. In particular, the serological level of sialylated carbohydrate antigens, which contain the sialic acid residue in their structure, showed effectiveness in diagnosing cancer behavior. Although large number of carbohydrate antigens in serum of cancer patients was elevated broadly in various cancers, each tumor marker has different sensitivity and specificity for each cancer. Therefore, the combined use of several tumor markers which have different characteristics is effective for better sensitivity in diagnosing cancer behavior. The mechanism of synthesizing cancerassociated carbohydrate antigens is not fully understood because it is very complex. In addition, new cancer-associated carbohydrate antigens are also identified by molecular oncological studies. Those investigations are considered to develop more effective tumor markers to diagnose cancer behavior.
\end{abstract}

Keywords: Tumor marker, sialylated carbohydrate antigens, clinical diagnosis

Glycoconjugates are important factors for various biological events in an organism. Aberrance of glycoconjugates, e.g. the change of structure or expression level, is detected in diseased patients and suggested to associate with the progression of diseases. In cancer, various aberrant glycoconjugates are expressed in cancerous tissues and investigated in relation to cancer behavior such as invasion and metastasis. Several kinds of those overexpressed glycoconjugates are detected in the serum of cancer patients and used as clinical diagnostic markers. The serological tumor markers are considered to be effective tools for screening cancer patients in routine medical care and monitoring the state of cancer patients.

In clinical tumor markers, there are many kinds of cancer associated carbohydrate antigens (Table 1). Each tumor marker has different sensitivity and specificity for various cancers. A large number of those carbohydrate antigens in serum of cancer patients is elevated broadly

\footnotetext{
*Address correspondence to:

Dr. Yoshinori Inagaki, Hepato-Biliary-Pancreatic Surgery Division, Department of Surgery, Graduate School of Medicine, The University of Tokyo, 7-3-1 Hongo, Bunkyo-ku, Tokyo 113-8655, Japan.

E-mail: yinagaki-tky@umin.ac.jp
}

in several gastrointestinal and gynecological cancers. Carbohydrate antigen (CA) 15-3 has been used for the evaluation of metastasis and recurrence of breast cancer although its sensitivity is poor $(1,2)$. CA125, whose epitope is on the tandem repeat peptide in MUC16, is suggested to be effective for longitudinal monitoring of ovarian cancer (3). Patients with cardiovascular disease are also suggested to show the elevation of CA125. In a study of patients with congestive heart failure, patients in a more advanced stage showed elevation of CA125 level (4). Therefore, CA125 shows a false positive if the patients suffered from those diseases. On the other hand, alpha-fetoprotein (AFP), which is frequently used as the diagnostic marker of hepatocellular carcinoma (HCC), also has carbohydrate structures. Elevation of AFP level also detects in benign liver diseases such as hepatitis and liver cirrhosis. The Lens culinaris agglutinin-reactive fraction of AFP (AFP-L3), which receives the structural change of carbohydrate (addition of fucose residue), contributes to an increase in sensitivity in screening HCC patients $(5,6)$. The peptide structure or glycoform of the epitope of these antigens have been investigated insufficiently.

Sialylated carbohydrate antigens, which contain sialic acid residues in their structure, frequently overexpress in cancer and are used for diagnosing cancer behavior. 
Table 1. Carbohydrate antigens that are used for clinical tumor marker

\begin{tabular}{lll}
\hline Tumor markers & Cancers which show significant elevation & References \\
\hline CA15-3 & Breast cancer & $(1,2)$ \\
CA125 & Ovarian cancer, Uterus cancer & $(3,27-29)$ \\
CA72-4 & Ovarian cancer, Uterus cancer, Gastric cancer & $(17-19)$ \\
CA19-9 & Colorectal cancer, Gastric cancer, Pancreatic cancer, Bile duct cancer & $(12-14,30)$ \\
sialy1 Le & Lung cancer, Gastric cancer, Bile duct cancer & $(31-33)$ \\
sialyl Tn & Ovarian cancer, Uterus cancer, Gastric cancer & $(16,32-34)$ \\
DUPAN-2 & Pancreatic cancer, Bile duct cancer & $(20-22)$ \\
SPan-1 & Pancreatic cancer, Bile duct cancer & $(35,36)$ \\
\hline
\end{tabular}

Many immunohistochemical studies showed the overexpression of sialylated carbohydrate antigens in cancer tissues by using lectins (7-10). Sialylation, the moiety of silalic acid, is considered to play an important role in tumor progression. Although various structures of sialylated carbohydrate are considered to be synthesized in cancer cells, specific types contribute to the clinical diagnosis for each kind of cancer. In the case of gastrointestinal cancers, sialyl Lewisrelated antigens such as sialyl Lewis $\mathrm{x}\left(\right.$ sialyl $\mathrm{Le}^{\mathrm{x}}$ ) and sialyl Lewis a (sialyl Le ${ }^{\mathrm{a}}$ ) antigens are the most often investigated tumor markers as sialylated carbohydrate antigens (11). Sialyl-Le ${ }^{\mathrm{a}}$ antigen is also known as CA 19-9 and frequently used in clinical diagnosis combined with Carcinoembryonic antigen (CEA) (1214). The combined evaluation of CEA and CA19-9 is recommended for better sensitivity in diagnosing the prognosis of gastrointestinal cancer patients. Another cancer-associated sialo-oligosaccharide antigen is sialyl $\mathrm{Tn}$ antigen. This antigen is barely expressed in normal epithelial tissue and is carried on cancer-associated proteins such as CD44 and mucins $(15,16)$. Those antigens are considered to have an important biological role in cancer invasion and metastasis. Serological levels of CA72-4, related to sialyl Tn antigen, are elevated in patients with gastric cancer and ovarian cancer (17-19). DUPAN-2, which is identical with sialyl $\mathrm{Le}^{\mathrm{c}}$ antigen, can be used for supportive diagnosis or monitoring behavior of pancreatic cancer (20-22). This antigen is also elevated in patients with hepato-biliary diseases but not with gastrointestinal cancers. Those sialylated carbohydrate antigens have been suggested to be related to induction of worse cancer behavior such as cancer cell invasion and metastasis while the detailed mechanism is still under investigation.

Mechanisms of overexpression of cancer-associated carbohydrate antigens are very complex. Up-regulation of glycosyltransferases was suggested to induce the aberrant structure of carbohydrate chains. There are many kinds of glycosyltransferases expressed in cancer cells, and therefore differences in up-regulated mechanisms caused by the different kinds of tumor markers for each cancer. In the case of sialic acidcontaining carbohydrate antigens, down-regulation of ST6GalNAc VI, which transfers $\alpha 2,6$-linked sialic acid to GlcNAc to synthesize a disialyl Lewis structure, is

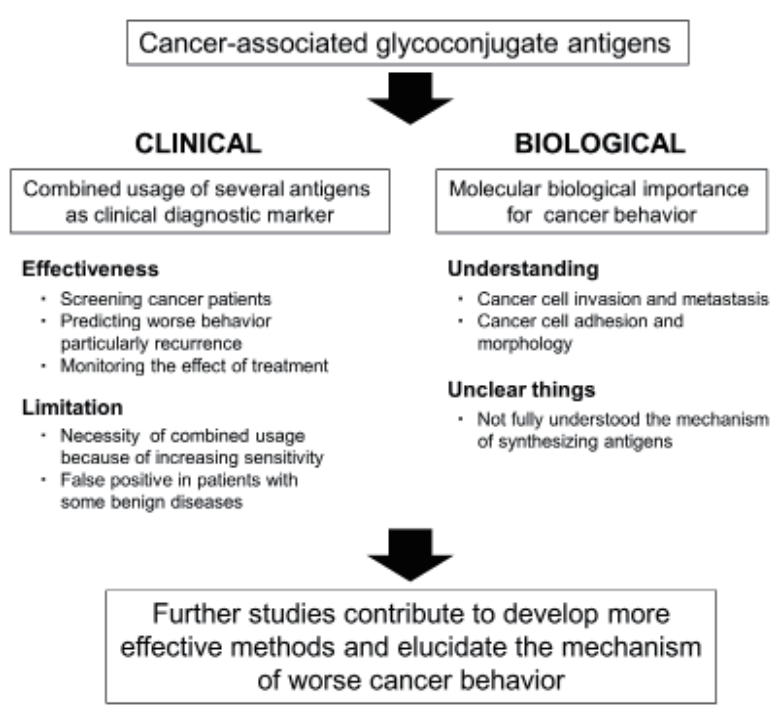

Figure 1. Current effectiveness and limitation of cancerassociated glycoocnjugate antigens.

suggested to induce the elevated levels of sialyl Lewisrelated antigens $(23,24)$. Biochemical and pathological studies performed by Marcos NT et al. suggested the main regulator of sialyl $\mathrm{Tn}$ antigen expression was ST6GalNAc-I activity $(25,26)$. Investigating the change of expression and activity of glycosyltransferases is considered to contribute to the elucidation of cancer biology including the expression of tumor markers.

Serological tumor markers have been used for monitoring the effect of surgical treatment or chemotherapy for cancer. If the level of tumor marker is elevated before the patient received the cancer therapy, an altered level of tumor marker is suggested to mean a change of cancer behavior. However, as described above, multiple tumor markers should be evaluated simultaneously to increase the sensitivity of diagnosis. Carbohydrate antigens which have different structures and specificity were identified. Therefore, effective use of those current tumor markers can contribute to a powerful diagnostic method while other imaging techniques are necessary for definitive diagnosis (Figure 1). In addition, new cancer-associated glycoconjugates can also be investigated for specific detection of cancers. Further investigation is expected to develop more sensitive and specific tools to screen cancer patients at an early stage. 


\section{References}

1. Park S, Ahn HK, Park LC, Hwang DW, Ji JH, Maeng CH, Cho SH, Lee JY, Park KT, Ahn JS, Park YH, Im YH. Implications of different CA 15-3 levels according to breast cancer subtype at initial diagnosis of recurrent or metastatic breast cancer. Oncology. 2012; 82:180-187.

2. Ideo H, Hinoda Y, Sakai K, Hoshi I, Yamamoto S, Oka M, Maeda K, Maeda N, Hazama S, Amano J, Yamashita K. Expression of mucin 1 possessing a 3'-sulfated core1 in recurrent and metastatic breast cancer. Int J Cancer. 2015. (doi:10.1002/ijc.29520)

3. Cramer DW, Bast RC Jr, Berg CD, Diamandis EP, Godwin AK, Hartge P, Lokshin AE, Lu KH, McIntosh MW, Mor G, Patriotis C, Pinsky PF, Thornquist MD, Scholler N, Skates SJ, Sluss PM, Srivastava S, Ward DC, Zhang Z, Zhu CS, Urban N. Ovarian cancer biomarker performance in prostate, lung, colorectal, and ovarian cancer screening trial specimens. Cancer Prev Res (Phila). 2011; 4:365-374.

4. Faggiano P, D'Aloia A, Brentana L, Bignotti T, Fiorina C, Vizzardi E, Dei Cas L. Serum levels of different tumour markers in patients with chronic heart failure. Eur J Heart Fail. 2005; 7:57-61.

5. Inagaki Y, Tang W, Makuuchi M, Hasegawa K, Sugawara Y, Kokudo N. Clinical and molecular insights into the hepatocellular carcinoma tumour marker des- $\gamma$ carboxyprothrombin. Liver Int. 2011; 31:22-35.

6. Song P, Gao J, Inagaki Y, Kokudo N, Hasegawa K, Sugawara Y, Tang W. Biomarkers: Evaluation of screening for and early diagnosis of hepatocellular carcinoma in Japan and china. Liver Cancer. 2013; 2:31-39.

7. Vierbuchen MJ, Fruechtnicht W, Brackrock S, Krause KT, Zienkiewicz TJ. Quantitative lectin-histochemical and immunohistochemical studies on the occurrence of alpha(2,3)- and alpha(2,6)-linked sialic acid residues in colorectal carcinomas. Relation to clinicopathologic features. Cancer. 1995; 76:727-735.

8. Tang W, Mafune K, Nakata M, Konishi T, Kojima N, Mizuochi T, Makuuchi M. Association of histochemical expression of Maackia amurensis leukoagglutinin-positive glycoconjugates with behaviour of human gastric cancer. Histopathology. 2003; 42:239-245.

9. Tang W, Guo Q, Usuda M, Kokudo N, Seyama Y, Minagawa M, Sugawara Y, Nakata M, Kojima N, Makuuchi M. Histochemical expression of sialoglycoconjugates in carcinoma of the papilla of Vater. Hepatogastroenterology. 2005; 52:67-71.

10. Inagaki Y, Tang W, Guo Q, Kokudo N, Sugawara Y, Karako H, Konishi T, Nakata M, Nagawa H, Makuuchi M. Sialoglycoconjugate expression in primary colorectal cancer and metastatic lymph node tissues. Hepatogastroenterology. 2007; 54:53-57.

11. Inagaki $\mathrm{Y}, \mathrm{Xu} \mathrm{H}$, Nakata $\mathrm{M}$, Seyama $\mathrm{Y}$, Hasegawa $\mathrm{K}$, Sugawara Y, Tang W, Kokudo N. Clinicopathology of sialomucin: MUC1, particularly KL-6 mucin, in gastrointestinal, hepatic and pancreatic cancers. Biosci Trends. 2009; 3:220-232.

12. Stiksma J, Grootendorst DC, van der Linden PW. CA 19-9 as a marker in addition to CEA to monitor colorectal cancer. Clin Colorectal Cancer. 2014; 13:239-244.

13. Lee EC, Yang JY, Lee KG, Oh SY, Suh YS, Kong SH, Yang HK, Lee HJ. The value of postoperative serum carcinoembryonic antigen and carbohydrate antigen 19-9 levels for the early detection of gastric cancer recurrence after curative resection. J Gastric Cancer. 2014; 14:221-228.
14. Reitz D, Gerger A, Seidel J, Kornprat P, Samonigg H, Stotz M, Szkandera J, Pichler M. Combination of tumour markers CEA and CA19-9 improves the prognostic prediction in patients with pancreatic cancer. J Clin Pathol. 2015. (doi:10.1136/jclinpath-2014-202451)

15. Singh R, Campbell BJ, Yu LG, Fernig DG, Milton JD, Goodlad RA, FitzGerald AJ, Rhodes JM. Cell surfaceexpressed Thomsen-Friedenreich antigen in colon cancer is predominantly carried on high molecular weight splice variants of CD44. Glycobiology. 2001; 11:587-592.

16. Byrd JC, Bresalier RS. Mucins and mucin binding proteins in colorectal cancer. Cancer Metastasis Rev. 2004; 23:7799.

17. Hamazoe R, Maeta M, Matsui T, Shibata S, Shiota S, Kaibara N. CA72-4 compared with carcinoembryonic antigen as a tumour marker for gastric cancer. Eur $\mathrm{J}$ Cancer. 1992; 28A:1351-1354.

18. Negishi Y, Iwabuchi H, Sakunaga H, Sakamoto M, Okabe $\mathrm{K}$, Sato H, Asano G. Serum and tissue measurements of CA72-4 in ovarian cancer patients. Gynecol Oncol. 1993; 48:148-154.

19. Ayude D, Rodríguez-Berrocal FJ, Ayude J, Blanco-Prieto S, Vázquez-Iglesias L, Vázquez-Cedeira M, Páez de la Cadena M. Preoperative serum CA 72.4 as prognostic factor of recurrence and death, especially at TNM stage II, for colorectal cancer. BMC Cancer. 2013;13:543.

20. Cooper EH, Forbes MA, Taylor M. An evaluation of DUPAN-2 in pancreatic cancer and gastrointestinal disease. Br J Cancer. 1990; 62:1004-1005.

21. Kawa S, Kato M, Oguchi H, Kobayashi T, Hsue GL, Koiwai T, Aoki Y, Furuta S, Kanai M. Elevated serum levels of Dupan-2 in pancreatic cancer patients negative for Lewis blood group phenotype. Scand J Gastroenterol. 1991; 26:1307-1318.

22. Okada K, Kawai M, Tani M, Hirono S, Miyazawa M, Shimizu A, Kitahata Y, Yamaue H. Predicting factors for unresectability in patients with pancreatic ductal adenocarcinoma. J Hepatobiliary Pancreat Sci. 2014; 21:648-653.

23. Tsuchida A, Okajima T, Furukawa K, Ando T, Ishida H, Yoshida A, Nakamura Y, Kannagi R, Kiso M, Furukawa $\mathrm{K}$. Synthesis of disialyl Lewis a $\left(\mathrm{Le}^{\mathrm{a}}\right)$ structure in colon cancer cell lines by a sialyltransferase, ST6GalNAc VI, responsible for the synthesis of alpha-series gangliosides. J Biol Chem. 2003; 278:22787-22794.

24. Senda M, Ito A, Tsuchida A, Hagiwara T, Kaneda T, Nakamura Y, Kasama K, Kiso M, Yoshikawa K, Katagiri Y, Ono Y, Ogiso M, Urano T, Furukawa K, Oshima S, Furukawa K. Identification and expression of a sialyltransferase responsible for the synthesis of disialylgalactosylgloboside in normal and malignant kidney cells: Downregulation of ST6GalNAc VI in renal cancers. Biochem J. 2007; 402:459-470.

25. Marcos NT, Pinho S, Grandela C, Cruz A, Samyn-Petit B, Harduin-Lepers A, Almeida R, Silva F, Morais V, Costa J, Kihlberg J, Clausen H, Reis CA. Role of the human ST6GalNAc-I and ST6GalNAc-II in the synthesis of the cancer-associated sialyl-Tn antigen. Cancer Res. 2004; 64:7050-7057.

26. Marcos NT, Bennett EP, Gomes J, et al. ST6GalNAc-I controls expression of sialyl-Tn antigen in gastrointestinal tissues. Front Biosci (Elite Ed) 2011; 3:1443-1455.

27. van der Burg ME, Lammes FB, Verweij J. The role of CA 125 in the early diagnosis of progressive disease in ovarian cancer. Ann Oncol. 1990; 1:301-302. 
28. Fader AN, Java J, Krivak TC, Bristow RE, Tergas AI, Bookman MA, Armstrong DK, Tanner EJ, Gershenson DM. The prognostic significance of pre- and post-treatment CA125 in grade 1 serous ovarian carcinoma: A gynecologic Oncology Group study. Gynecol Oncol. 2014; 132:560-565.

29. Kotowicz B, Kaminska J, Fuksiewicz M, Kowalska M, Jonska-Gmyrek J, Gawrychowski K, Sobotkowski J, Skrzypczak M, Starzewski J, Bidzinski M. Clinical significance of serum CA-125 and soluble tumor necrosis factor receptor type I in cervical adenocarcinoma patients. Int J Gynecol Cancer. 2010; 20:588-592.

30. Kondo N, Murakami Y, Uemura K, Sudo T, Hashimoto Y, Sasaki H, Sueda T. Elevated perioperative serum CA 19-9 levels are independent predictors of poor survival in patients with resectable cholangiocarcinoma. J Surg Oncol. 2014; 110:422-429.

31. Satoh H, Ishikawa H, Kamma H, Yamashita YT, Takahashi H, Ohtsuka M, Hasegawa S. Serum sialyl lewis X-i antigen levels in non-small cell lung cancer: Correlation with distant metastasis and survival. Clin Cancer Res. 1997; 3:495-499.

32. Nakagoe T, Sawai T, Tsuji T, Jibiki MA, Nanashima A, Yamaguchi H, Yasutake T, Ayabe H, Arisawa K, Ishikawa $\mathrm{H}$. Predictive factors for preoperative serum levels of sialy
Lewis(x), sialyl Lewis(a) and sialyl Tn antigens in gastric cancer patients. Anticancer Res. 2002; 22:451-458.

33. Inagaki Y, Gao J, Song P, Kokudo N, Nakata M, Tang W. Clinicopathological utility of sialoglycoconjugates in diagnosing and treating colorectal cancer. World J Gastroenterol. 2014; 20:6123-6132.

34. Inoue $M$, Fujita $M$, Nakazawa $A$, Ogawa $H$, Tanizawa O. Sialyl-Tn, sialyl-Lewis Xi, CA 19-9, CA 125 , carcinoembryonic antigen, and tissue polypeptide antigen in differentiating ovarian cancer from benign tumors. Obstet Gynecol. 1992; 79:434-440.

35. Kiriyama S, Hayakawa T, Kondo T, Shibata T, Kitagawa M, Ono H, Sakai Y. Usefulness of a new tumor marker, Span1 , for the diagnosis of pancreatic cancer. Cancer. 1990; 65:1557-1561.

36. Tsutsumi K, Kawamoto H, Hirao K, Sakakihara I, Yamamoto N, Noma Y, Fujii M, Kato H, Ogawa T, Ishida E, Kuwaki K, Nouso K, Okada H, Yamamoto K. A novel scoring system for arterial invasion of pancreatic body and tail cancer based on multidetector row computed tomography and biomarkers. Pancreatology. 2013; 13:161169.

(Received April 10, 2015; Accepted April 27, 2015) 